\title{
Pediatric Sinonasal Undifferentiated Carcinoma: Case Report and Literature Review
}

\author{
Jefferson R. Wilson, Shobhan Vachhrajani, Jennifer Li, Melody Sun, Cynthia Hawkins, \\ James T. Rutka
}

Can. J. Neurol. Sci. 2010; 37: 873-877

Sinonasal undifferentiated carcinoma (SNUC) is a rare, aggressive malignancy arising from the nasal cavity and paranasal sinuses. Sinonasal undifferentiated carcinoma was first reported in 1986 by Frierson et al, and since then less than 100 cases have been reported in the literature. ${ }^{1}$ One previous pediatric case has been described in a 12-year-old boy in India. ${ }^{2}$ Sinonasal undifferentiated carcinoma is histologically classified as part of a spectrum of neuroendocrine carcinomas originating from the Schneiderian epithelium or the nasal ectoderm in the paranasal sinuses. ${ }^{1}$ Here, we report only the second case of SNUC to arise in childhood involving a teenager with disease arising from the nasal cavity and extending through the paranasal air sinuses into the anterior cranial fossa and brain.

\section{Case Report}

A 15-year-old, previously healthy male presented with a two week history of increasingly severe frontal headaches, nausea, vomiting and fatigue. Family members noted disinhibition of speech and atypical behaviour occurring in the month prior to presentation. His neurological examination revealed bilateral papilledema with no other focal deficits. All bloodwork was normal, and serum germ cell tumor markers were also negative. Computed tomogram (CT) and magnetic resonance imaging (MRI) scan of the brain and paranasal air sinuses revealed a 5.5 x 4.4 x $5.6 \mathrm{~cm}$ right sided mass arising from the nasal cavity, ethmoid and sphenoid sinuses. It had eroded through the cribriform plate into the anterior cranial fossa. The mass was not calcified on CT scan (Figure 1), and isodense to brain with heterogeneous contrast enhancement. The mass was isointense to brain on T1 and T2 weighted MR images (Figures 2, 3, 4) and demonstrated heterogeneous enhancement with gadolinium. Surrounding vasogenic cerebral edema and mass effect were noted. No evidence of metastasis was seen on MRI of the spine.

\section{Operative Procedure}

A combined endonasal endoscopic and bifrontal craniotomy surgical plan was devised to treat this lesion. Initially, a right endoscopic transnasal approach was taken to obtain tissue for pathologic diagnosis. After obtaining the diagnosis of SNUC from the biopsy, the patient was brought back to the operating room five days later for resection of the remaining tumor located intracranially and in the paranasal sinuses. A bifrontal craniotomy, centered to the right of midline was fashioned. Dissection through the anterior interhemispheric fissure from the right side was undertaken until the tumor was encountered about $4 \mathrm{~cm}$ above the floor of the anterior cranial fossa. The lesion was

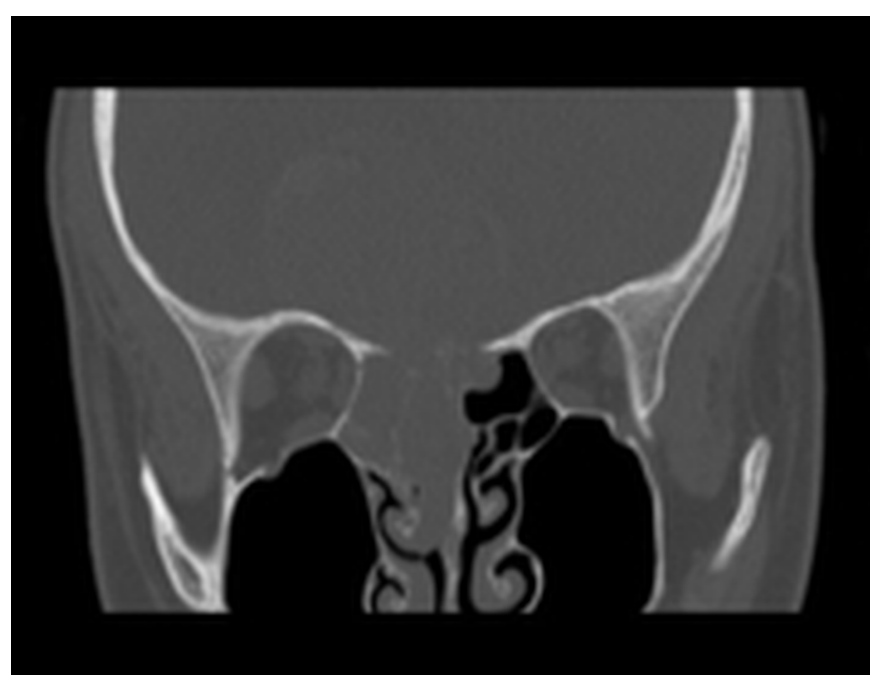

Figure 1: Bone window of a coronal CT scan showing tumor arising from the right nasal cavity with extension into the ethmoid sinus and through the cribriform plate into the anterior cranial fossa. Note the lack of calcification within the tumor mass.

highly vascular and was removed with suction and the ultrasonic aspirator following the development of planes around the tumor at the interface with the normal brain. Upon reaching the skull base, the mass was visualized eroding through the cribriform plate into the ethmoid sinuses. The residua of the tumor in the nasal sinuses was resected from above and below using the endoscope. An on-lay split-thickness frontal cranial bone graft was used to repair the hole in the anterior cranial base. A vascularized pericranial tissue flap was then used to support the closure of the dural defect supplemented by concentrated fibrin tissue glue.

Histologic examination revealed solid sheets of poorly differentiated cells with high nuclear to cytoplasmic ratio,

From the Divisions of Neurosurgery and Neuropathology, Hospital for Sick Children, University of Toronto, Toronto, Ontario, Canada.

Received February 9, 2010. Final Revisions Submitted April 20, 2010. Correspondence to: James T. Rutka, Division of Neurosurgery, The University of Toronto, The Hospital for Sick Children, 555 University Avenue, Suite 1503, Toronto, Ontario, M5G 1X8, Canada. 


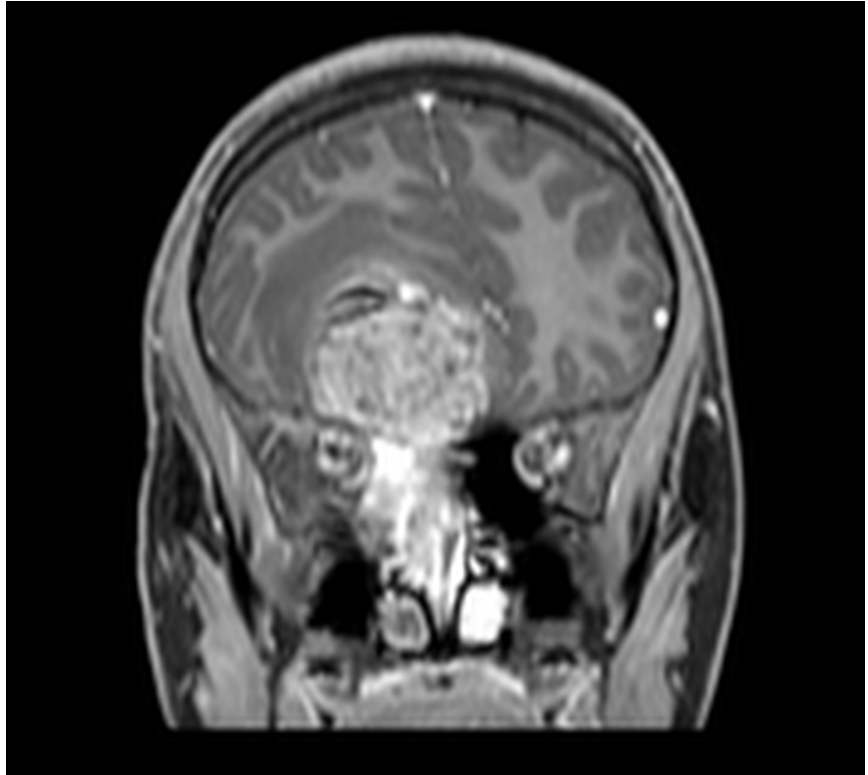

Figure 2: Coronal T1-weighted gadolinium enhanced MRI scan confirming the findings of a large tumor mass arising in the nasal cavity, and extending into the anterior cranial fossa. Mass effect on the inferior frontal lobe and minimal midline shift is evident.

broadly infiltrating trabeculae and vesicular nuclei were noted with moderate mitotic activity (Figure 5). There were prominent areas of apoptosis and necrosis visualized as well as a vascularized tumor stroma with regions of fibro-myxoid change. Immunohistochemistry showed positive staining for vimentin and pan cytokeratin (Figure 6). In situ hybridization for Epstein Barr Virus (EBV) was negative. Electron microscopy showed a mixture of primitive small cells as well as larger epithelial cells with well formed long intercellular junctions. A diagnosis of SNUC was made.

Postoperatively, he remained neurologically unchanged and experienced significant reduction in headaches prior to being discharged home on the tenth postoperative day. Magnetic resonance imaging was performed two days after surgery demonstrated gross total tumor resection. He received adjuvant fractionated radiotherapy, consisting of $6000 \mathrm{cGy}$ in 30 fractions delivered to the tumor bed and surrounding margin. Concurrent cisplatinum chemotherapy was also administered. Twelve months following surgery, the patient remains neurologically well with no radiographic or clinical evidence of tumor recurrence (Figures 7,8).

\section{Discussion}

An aggressive type of neuroendocrine carcinoma, sinonasal undifferentiated carcinoma (SNUC) is a malignant lesion arising from the epithelium of the nasal cavity and paranasal air sinuses rarely occurring in the pediatric population. It is most frequently diagnosed in the sixth decade of life with a higher prevalence in males. ${ }^{1,-5}$ Patients are typically asymptomatic early in the course of their disease despite extensive spread, largely owing to continued tumor growth through the "silent anatomical spaces" of the nasal cavity and paranasal air sinuses. ${ }^{6}$

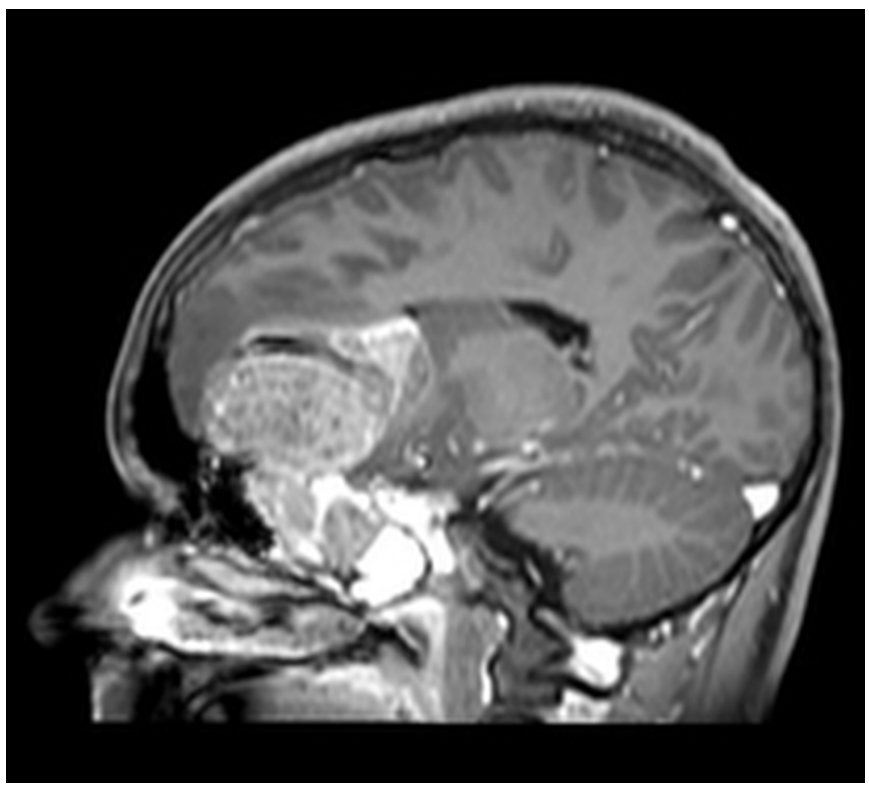

Figure 3: Sagittal T1-weighted gadolinium enhanced MRI scan displaying erosion of the tumor mass from the paranasal sinuses into the anterior cranial fossa.

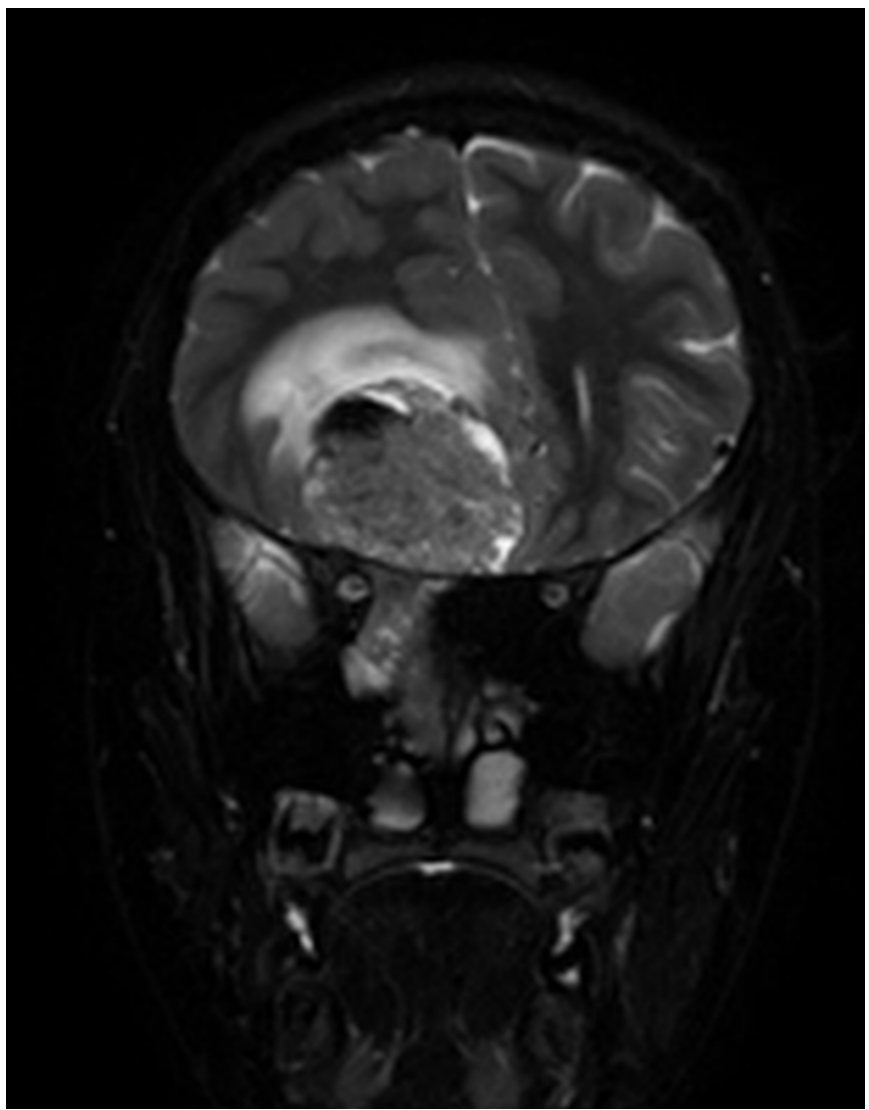

Figure 4: Coronal T2-weighted MRI scan confirming the heterogeneous solid and cystic nature of the intracranial tumor component. Perilesional edema is clearly evident as well as tumor-associated midline shift. 


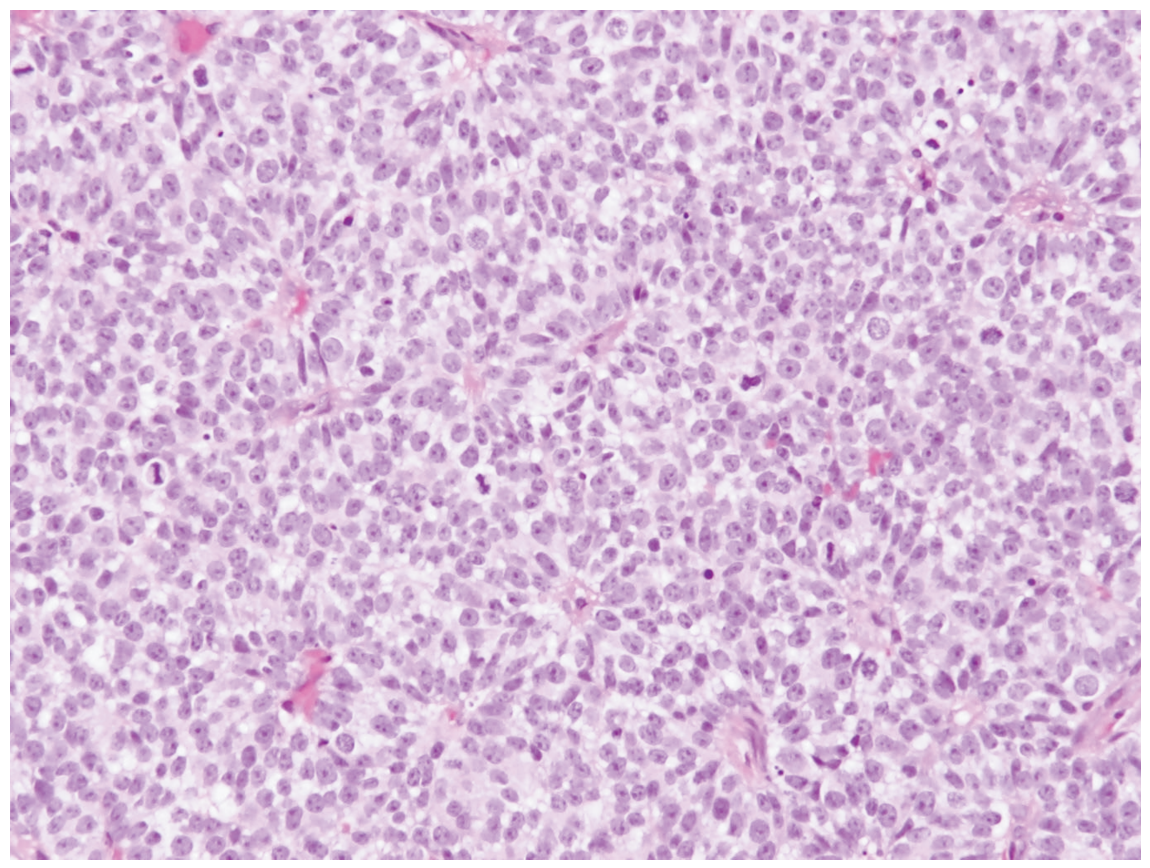

Figure 5: Photomicrograph of $H \& E$ slide at $40 X$ magnification showing solid sheets of vesicular nuclei, high nuclear to cytoplasmic ratio, moderate mitotic activity, and prominent areas of apoptosis and necrosis.

Most commonly, patients present with symptoms suggestive of benign sinus disease including bloody rhinorrhea, facial pain, and nasal obstruction..$^{3,7,8}$ The interval between onset of symptoms and diagnosis is approximately four months..$^{3,9}$ In contrast to most cases reported in the literature, our patient presented with clinical stigmata of raised intracranial pressure (ICP), and behavioural changes that may be explained by progressive frontal lobe dysfunction due to tumor compression. Such presentation is similar to the only other reported pediatric case of SNUC; that patient also displayed signs of increased ICP and frontal lobe involvement without clinical evidence of nasal or orbital involvement. ${ }^{2}$ Tumor metastasis, occurring via hematogenous spread, is rare but when present commonly involves the lungs and bone. There are infrequent reports of tumor seeding the cerebrospinal fluid leading to spinal drop metastases visible on spinal MRI. ${ }^{10}$

Sinonasal undifferentiated carcinoma is difficult to distinguish radiologically from other aggressive lesions of the anterior skull base and paranasal sinuses. The differential diagnosis includes squamous cell carcinoma, lymphoma, esthesioneuroblastoma, melanoma, neuroendocrine carcinomas and in the pediatric population, rhabdomyosarcoma. On imaging, SNUC is characteristically large, often measuring greater than 4 $\mathrm{cm}$ in greatest dimension, and expansile with focal bony destruction and invasion into surrounding soft tissue. ${ }^{11}$ Computed tomogram scan typically demonstrates a non-calcified mass that erodes through bone and exhibits heterogeneous contrast enhancement. On MRI, lesions are isointense on T1weighted images and iso- to hyperintense on T2-weighted images. Tumors enhance heterogeneously with gadolinium.
Histologically, SNUC appears as nests, sheets, and trabeculae of small to medium sized cells with large, ovoid nuclei and eosinophilic cytoplasm. ${ }^{1}$ Tumors generally exhibit signs of aggressive behaviour including a high mitotic rate, prominent vascular permeation, and well defined areas of necrosis. ${ }^{1}$ Immunohistochemical staining has proven useful to help distinguish SNUC from other types of sinonasal and anterior skull base tumors. Cerilli et al characterized the immunohistochemical profile in SNUC tumor specimens from 25 patients and found the following staining patterns: Ki-67 95\% positive; p53 59\% positive; CD-99 14\% positive; epithelial membrane antigen $18 \%$ positive; and neuron specific enolase $18 \%$ positive. ${ }^{6}$ In general SNUC stains negatively for chromogranin, synaptophysin, placental alkaline phosphatase and carcinoembryonic antigen. ${ }^{6}$

Certain workplace exposures such as nickel refining and soft wood processing have been associated with this and other malignant sinonasal tumors. ${ }^{12}$ The relationship between SNUC and smoking remains unclear. Frierson et al reported that seven of eight patients in their series had a significant history of cigarette smoking, whereas Righi et al reported that only one in seven patients in their series had similar exposure. ${ }^{1,13}$ Our patient had no relevant occupational exposures and denied ever having smoked. Adult survivors of heritable retinoblastoma have been implicated to be at an elevated risk for the development of SNUC. ${ }^{14,15}$ These patients carry a germline mutation at one of the two homologous RB-1 gene loci on chromosome 13 and are at an increased risk later in life for development of non-ocular ectodermal tumors including small cell sinonasal tumors. Epstein Barr Virus has been implicated as a potential pathogen in 


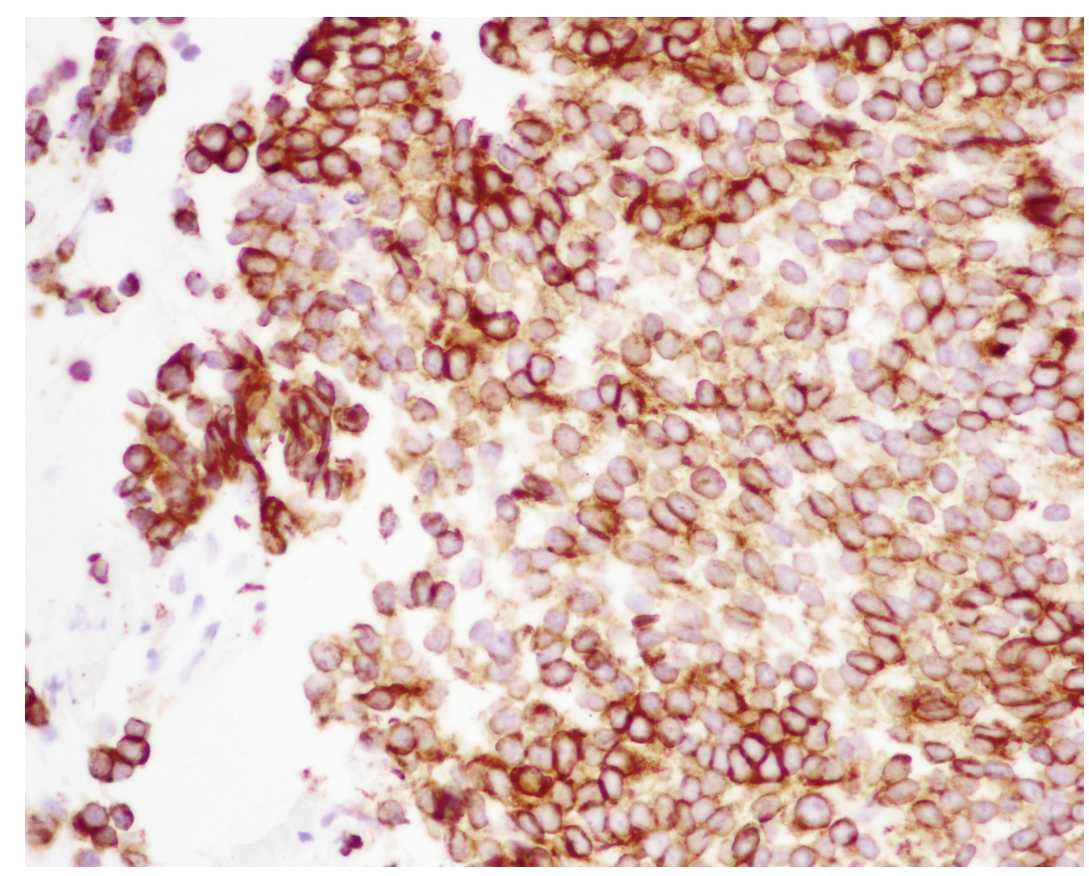

Figure 6: Photomicrograph at $40 X$ magnification showing diffuse positive immunohistochemical staining for pancytokeratin.

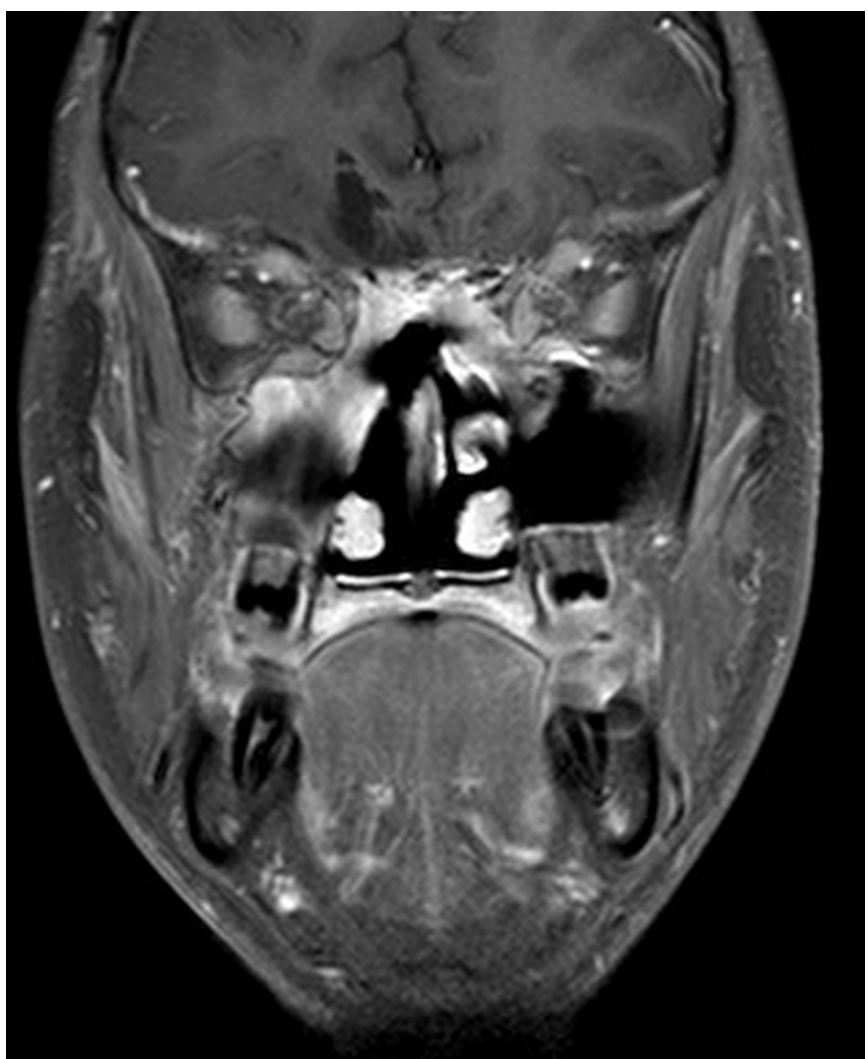

Figure 7: Coronal T1-weighted gadolinium enhanced MRI scan showing gross total resection of intranasal and intracranial tumor components. Note the resolution of mass effect on the frontal lobe and lack of contrast enhancement in the tumor bed. the development of SNUC as several studies have demonstrated that $30-60 \%$ of specimens tested were positive for EBV using insitu hybridization. ${ }^{16}$ However in a more recent study using in situ hybridization for EBER-1, Jeng et al demonstrated that strictly defined SNUC is not associated with EBV and that previously reported EBV-positive undifferentiated carcinomas of the sinonasal region likely comprised primary sinonasal nasopharyngeal type undifferentiated carcinoma. ${ }^{17}$

Due to the paucity of cases, no consensus exists for the treatment of SNUC. Current literature favors a multimodality approach that involves a combination of surgical resection, adjuvant radiotherapy and chemotherapy. ${ }^{18}$ Initial studies in which SNUC was treated with radiation alone or with a combination of radiation and chemotherapy reported dismal patient outcomes with mean survival of one year. ${ }^{8,13}$ More recent studies evaluating the efficacy of surgery combined with either neoadjuvant or adjuvant chemoradiation have yielded more optimistic results. In one of the largest case series involving 19 adult patients treated at the University of Virginia, the standard treatment regimen included induction chemotherapy (cisplatin and etoposide or cyclophosphamide, doxorubicin and vincristine) followed by pre-operative radiation therapy (50005400 cGy in 25 fractions) and surgical resection. ${ }^{8}$ Patients in poor health or those harbouring tumors deemed to be surgically unresectable underwent palliative radiation or chemoradiation. The two-year survival rate for the ten patients treated with chemoradiation followed by surgery was $64 \%$ as compared to $25 \%$ for the nine patients who were treated non-operatively.

In the only other reported pediatric case in the literature, treatment consisted of gross total resection followed by postoperative radiotherapy. ${ }^{2}$ The patient was noted to be 


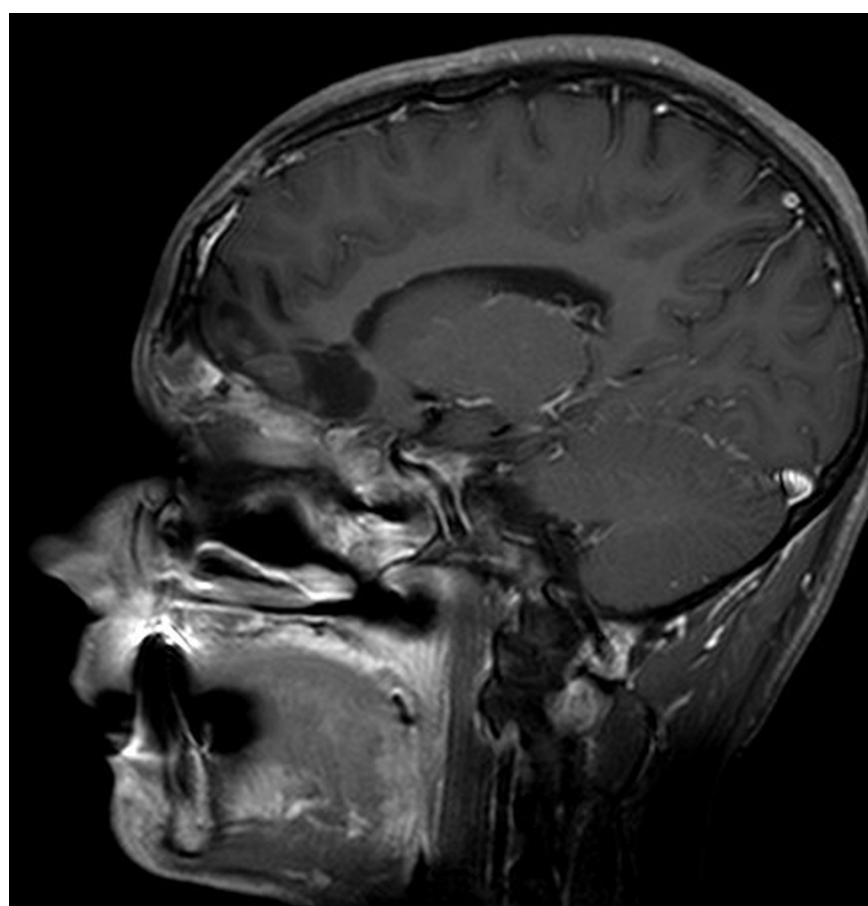

Figure 8: Sagittal T1-weighted gadolinium enhanced MRI scan showing gross total resection of tumor from the paranasal air sinuses and anterior cranial fossa. Note the lack of mass effect on the frontal lobe and lack of contrast enhancement in the tumor cavity.

asymptomatic at six months post diagnosis. Our patient received similar treatment with additional chemotherapy and has no clinical or radiographic evidence of tumor recurrence six months after diagnosis. Our case likely represents a rare, sporadic pediatric case of SNUC with as yet unclear etiology and pathogenesis. Arguably the reporting of more cases will aid in elucidating etiological associations.

We present a rare pediatric case of SNUC with symptomatic intracranial extension. To our knowledge, this is only the second pediatric case of SNUC reported to date and its pathogenesis remains elusive. The treatment of these tumors remains a challenge due to the rarity of diagnosis. In order to achieve local disease control and maximize survival, we recommend a multimodal approach to treatment including radical surgical resection accompanied by adjuvant or neoadjuvant chemotherapy or radiotherapy. The rarity of SNUC means that multicentre efforts will be required to determine the optimal treatment for this disease.

\section{REFERENCES}

1. Frierson HF, Jr., Mills SE, Fechner RE, Taxy JB, Levine PA. Sinonasal undifferentiated carcinoma. An aggressive neoplasm derived from schneiderian epithelium and distinct from olfactory neuroblastoma. Am J Surg Pathol. 1986 Nov;10(11):771-9.

2. Kumar R, Chandra A, Rastogi A. Intracranial sinonasal undifferentiated carcinoma (SNUC) in a child. Childs Nerv Syst. 2006 Sep;22(9):1208-11.

3. Gallo O, Graziani P, Fini-Storchi O. Undifferentiated carcinoma of the nose and paranasal sinuses. An immunohistochemical and clinical study. Ear Nose Throat J. 1993 Sep;72(9):588-90, 593-5.

4. Rischin D, Porceddu S, Peters L, Martin J, Corry J, Weih L. Promising results with chemoradiation in patients with sinonasal undifferentiated carcinoma. Head Neck. 2004 May;26(5): 435-41.

5. Rosenthal DI, Barker JL, Jr., El-Naggar AK, et al. Sinonasal malignancies with neuroendocrine differentiation: patterns of failure according to histologic phenotype. Cancer. 2004 Dec 1; 101(11):2567-73

6. Cerilli LA, Holst VA, Brandwein MS, Stoler MH, Mills SE. Sinonasal undifferentiated carcinoma: immunohistochemical profile and lack of EBV association. Am J Surg Pathol. 2001 Feb;25(2):156-63.

7. Pitman KT, Lassen LF. Pathologic quiz case 2. Sinonasal undifferentiated carcinoma (SNUC). Arch Otolaryngol Head Neck Surg. 1995 Oct;121(10):1201, 3 .

8. Levine PA, Frierson HF, Jr, Stewart FM, Mills SE, Fechner RE, Cantrell RW. Sinonasal undifferentiated carcinoma: a distinctive and highly aggressive neoplasm. Laryngoscope. 1987 Aug;97(8 Pt 1):905-8.

9. Musy PY, Reibel JF, Levine PA. Sinonasal undifferentiated carcinoma: the search for a better outcome. Laryngoscope. 2002 Aug;112(8 Pt 1):1450-5.

10. Ghosh S, Weiss M, Streeter O, Sinha U, Commins D, Chen TC. Drop metastasis from sinonasal undifferentiated carcinoma: clinical implications. Spine. 2001 Jul 1;26(13):1486-91.

11. Phillips CD, Futterer SF, Lipper MH, Levine PA. Sinonasal undifferentiated carcinoma: CT and MR imaging of an uncommon neoplasm of the nasal cavity. Radiology. 1997 Feb; 202(2):477-80

12. Sunderman FW, Jr., Morgan LG, Andersen A, Ashley D, Forouhar FA. Histopathology of sinonasal and lung cancers in nickel refinery workers. Ann Clin Lab Sci. 1989 Jan-Feb;19(1):44-50.

13. Righi PD, Francis F, Aron BS, Weitzner S, Wilson KM, Gluckman J. Sinonasal undifferentiated carcinoma: a 10-year experience. Am J Otolaryngol. 1996 May-Jun;17(3):167-71.

14. Greger V, Schirmacher P, Bohl J, et al. Possible involvement of the retinoblastoma gene in undifferentiated sinonasal carcinoma. Cancer. 1990 Nov 1;66(9):1954-9.

15. Frierson HF, Jr, Ross GW, Stewart FM, Newman SA, Kelly MD. Unusual sinonasal small-cell neoplasms following radiotherapy for bilateral retinoblastomas. Am J Surg Pathol. 1989 Nov;13 (11):947-54

16. Gallo O, Di Lollo S, Graziani P, Gallina E, Baroni G. Detection of Epstein-Barr virus genome in sinonasal undifferentiated carcinoma by use of in situ hybridization. Otolaryngol Head Neck Surg. 1995 Jun;112(6):659-64.

17. Jeng YM, Sung MT, Fang CL, et al. Sinonasal undifferentiated carcinoma and nasopharyngeal-type undifferentiated carcinoma: two clinically, biologically, and histopathologically distinct entities. Am J Surg Pathol. 2002 Mar;26(3):371-6.

18. Mendenhall WM, Mendenhall CM, Riggs CE, Jr., Villaret DB, Mendenhall NP. Sinonasal undifferentiated carcinoma. Am J Clin Oncol. 2006 Feb;29(1):27-31. 